\title{
Constructing Signals With Prescribed Properties
}

\author{
Yonina C. Eldar, Member, IEEE
}

\begin{abstract}
This letter introduces a general framework for constructing signals with prescribed properties that can be described as inner products of the signal with a set of vectors, with almost arbitrary construction and constraint spaces. We first derive a procedure for constructing a signal in a given construction space, with prescribed properties in a given constraint space. Building upon these results, we develop a procedure for constructing a signal with prescribed properties in two disjoint constraint spaces.
\end{abstract}

Index Terms-Consistent sampling, signal construction.

\section{INTRODUCTION}

$\mathbf{T}$ HERE are various contexts in which it may be desirable to construct a signal with prescribed properties. In this letter we consider the case in which these properties can be described as inner products of the signal with a set of vectors.

Specifically, it may be desirable to construct a signal $f$ to lie in a construction space $\mathcal{W}$, and to have some additional properties that can be described as inner products of $f$ with vectors $s_{i}$ that span a constraint space $\mathcal{S}$. For example, we may wish to construct a bandlimited signal with prescribed values over a given time interval. It may also be desirable to construct a signal with prescribed properties in two disjoint constraint spaces $\mathcal{S}$ and $\mathcal{W}$. For example, we may wish to construct a signal with prescribed lowpass coefficients and prescribed values over a time interval. In this letter, we develop a general framework for constructing signals of this form.

We denote by $P_{\mathcal{V}}$ the orthogonal projection onto the subspace $\mathcal{V}$, and by $(\cdot)^{*}$ the adjoint of the corresponding transformation. The inner product between vectors $x, y \in \mathcal{H}$ is denoted by $\langle x, y\rangle=x^{*} y$.

\section{Constructing Signals in $\mathcal{W}$ With PRescribed PROPERTIES IN $\mathcal{S}$}

Suppose we wish to construct a signal $f$ to lie in a subspace $\mathcal{W}$ of a Hilbert space $\mathcal{H}$, and to have some additional properties in a subspace $\mathcal{S} \subseteq \mathcal{H}$ that can be described as constraints of the form $\left\langle s_{i}, f\right\rangle=c[i]$ for some $c \in l_{2}$, where the vectors $s_{i}$

\footnotetext{
Manuscript received January 10, 2002; revised July 24, 2002. This work is supported in part by BAE Systems Cooperative Agreement RP6891 under Army Research Laboratory Grant DAAD19-01-2-0008, by the Army Research Laboratory Collaborative Technology Alliance through BAE Systems Subcontract RK78554, and by Texas Instruments through the TI Leadership University Consortium. The associate editor coordinating the review of this manuscript and approving it for publication was Prof. Steven Kay.

The author was with the Research Laboratory of Electronics, Massachusetts Institute of Technology, Cambridge, MA 02139 USA. She is now with the Technion, Israel Institute of Technology, Haifa 32000, Israel (e-mail: yonina@ee.technion.ac.il).

Digital Object Identifier 10.1109/LSP.2002.807870
}

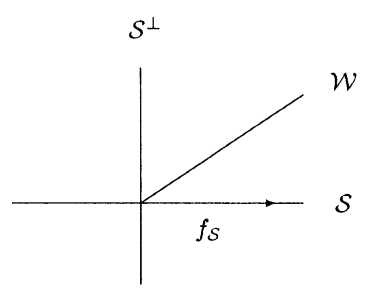

(a)

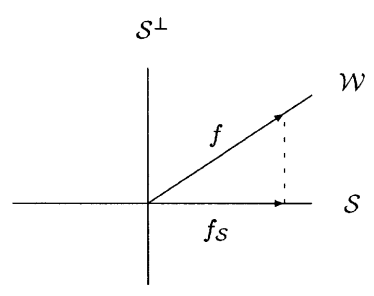

(b)
Fig. 1. Illustration of the construction of $f \in \mathcal{W}$ with a given orthogonal projection $f_{\mathcal{S}}$ onto $\mathcal{S}$ with $\mathcal{H}=\mathcal{W} \oplus \mathcal{S}^{\perp}$. (a) Desired orthogonal projection onto $\mathcal{S}$. (b) Unique signal $f \in \mathcal{W}$ with the given projection.

form a Riesz basis ${ }^{1}$ [1] for $\mathcal{S}$. We do not require that $\mathcal{S}=\mathcal{W}$; however, we assume that $\operatorname{dim} \mathcal{S}=\operatorname{dim} \mathcal{W}$. This ensures that the number of degrees of freedom in constructing $f$ is equal to the number of constraints ${ }^{2}$ on $f$. Since for any $f \in \mathcal{W} \cap \mathcal{S}^{\perp}$, $\left\langle s_{i}, f\right\rangle=0$, we further assume that $\mathcal{W}$ and $\mathcal{S}^{\perp}$ are disjoint, i.e., $\mathcal{W} \cap \mathcal{S}^{\perp}=\{0\}$. If $\mathcal{W}$ and $\mathcal{S}$ have finite dimension, then this implies that $\mathcal{H}=\mathcal{W} \oplus \mathcal{S}^{\perp}$. We therefore explicitly assume that $\mathcal{H}=\mathcal{W} \oplus \mathcal{S}^{\perp}$.

Since $f \in \mathcal{W}, f=\sum_{i} d[i] w_{i}$ for some $d \in l_{2}$, where the vectors $w_{i}$ from a Riesz basis for $\mathcal{W}$. With $S$ and $W$ denoting the set transformations ${ }^{3}$ corresponding to $s_{i}$ and $w_{i}$ respectively, our problem is to find $d$ such that $f=W d$ satisfies

$$
S^{*} f=S^{*} W d=c .
$$

To this end, we first develop a geometric interpretation of our problem. Denoting by $f_{\mathcal{S}}=P_{\mathcal{S}} f$ the orthogonal projection of $f$ onto $\mathcal{S},\left\langle s_{i}, f\right\rangle=\left\langle P_{\mathcal{S}} s_{i}, f\right\rangle=\left\langle s_{i}, P_{\mathcal{S}} f\right\rangle=\left\langle s_{i}, f_{\mathcal{S}}\right\rangle$. Furthermore, since $f_{\mathcal{S}} \in \mathcal{S}$ and the vectors $s_{i}$ span $\mathcal{S}, f_{\mathcal{S}}$ is uniquely determined by $c[i]=\left\langle s_{i}, f\right\rangle=\left\langle s_{i}, f_{\mathcal{S}}\right\rangle$. Therefore, constructing $f$ to have prescribed inner products $c[i]$ is equivalent to constructing $f$ to have a prescribed projection $f_{\mathcal{S}}$. Geometrically, our problem reduces to constructing $f \in \mathcal{W}$ such that its orthogonal projection onto $\mathcal{S}$ is equal to some given projection $f_{\mathcal{S}}$.

Fig. 1(a) depicts the desired projection $f_{\mathcal{S}}=P_{\mathcal{S}} f$ of an unknown signal $f \in \mathcal{W}$. The problem is to construct $f$ with this projection. Since the direction of $\mathcal{W}$ is known, there is a unique vector in $\mathcal{W}$ whose projection onto $\mathcal{S}$ is $f_{\mathcal{S}}$; this vector is illustrated in Fig. 1(b).

\footnotetext{
${ }^{1} \mathrm{~A}$ Riesz basis is a basis with some additional norm constraints that guarantee, for example, that $c$ is in $l_{2}$. In particular, any basis for a finite-dimensional space is a Riesz basis.

${ }^{2}$ If $\operatorname{dim} \mathcal{S}>\operatorname{dim} \mathcal{W}$, then in general we will not be able to construct such a signal $f$, since there are more constraints than degrees of freedom. If $\operatorname{dim} \mathcal{S}<$ $\operatorname{dim} \mathcal{W}$, then in general $f$ will not be unique.

${ }^{3}$ A set transformation corresponding to $\left\{x_{i} \in \mathcal{H}\right\}$ is defined by $X a=$ $\sum_{i} a[i] x_{i}$ for $a \in l_{2}$. From the definition of the adjoint, if $a=X^{*} y$ then $a[i]=\left\langle x_{i}, y\right\rangle$.
} 
Fig. 1 demonstrates that with $\mathcal{H}=\mathcal{W} \oplus \mathcal{S}^{\perp}$, we can always construct an $f \in \mathcal{W}$ such that $S^{*} f=c$. To explicitly construct $f$, we rely on the following theorem [2].

Theorem 1: Let the vectors $\left\{w_{i}\right\}$ corresponding to $W$ and the vectors $\left\{s_{i}\right\}$ corresponding to $S$ form Riesz bases for $\mathcal{W} \subseteq \mathcal{H}$ and $\mathcal{S} \subseteq \mathcal{H}$, respectively, with $\mathcal{H}=\mathcal{W} \oplus \mathcal{S}^{\perp}$. Then $S^{*} W$ is invertible.

From Theorem 1, the unique $d$ satisfying (1) is $d=\left(S^{*} W\right)^{-1} c$, and the unique $f \in \mathcal{W}$ such that $S^{*} f=c$ is

$$
f=W\left(S^{*} W\right)^{-1} c
$$

By choosing different spaces $\mathcal{H}, \mathcal{W}$, and $\mathcal{S}$ and using (2), we can develop a variety of interesting constructions.

We note that our problem can be formulated as a consistent reconstruction problem [2]-[5], in which it is desired to reconstruct $f$ using vectors $w_{i}$ from measurements $c[i]=\left\langle s_{i}, f\right\rangle$, where the reconstructed signal is required to yield the same measurements as $f$. Using results obtained in this context [2], [4], [5], we may extend (2) to include the case of linearly dependent vectors $s_{i}$.

\section{Constructing A Time-Limited Sequence With PRESCRIBED LOWPASS COEFFICIENTS}

To illustrate the details of (2), we consider an application to the construction of a time-limited sequence with prescribed lowpass coefficients. Thus, $\mathcal{H}$ is the space of sequences $x[k]=0$ for $k<0, k \geq n$, and $\mathcal{W}$ is the space of sequences $x[k]=0$ for $k<0, k \geq m$ where $m=2 m^{\prime}+1<n$. We wish to construct $f \in \mathcal{W}$ such that the $m$ lowpass discrete Fourier transform (DFT) coefficients of the $n$-point DFT $F[k], 0 \leq k \leq n-1$ of $f$ are equal to some prescribed values $c[i]$.

To construct $f$ we first determine vectors $s_{i}$ such that $\left\langle s_{i}, f\right\rangle=c[i], 0 \leq i \leq m-1$. Specifically, $s_{i}[k]=e^{j 2 \pi\left(i-m^{\prime}\right) k / n}$ for $0 \leq k \leq n-1$ and zero otherwise. Then, $\mathcal{S}$ is the space of bandlimited sequences $x[k]$ such that $X[k]=0, m^{\prime}<k<n-m^{\prime}$, where $X[k]$, $0 \leq k \leq n-1$ denotes the $n$-point DFT of $x[k]$.

To apply (2), we select the basis $w_{i}[k]=\delta[i-k], 0 \leq i \leq$ $m-1$ for $\mathcal{W}$. We next compute $d=\left(S^{*} W\right)^{-1} c$; the details are given in [2]. Then $f[k]=\sum_{i=0}^{n-1} w_{i}[k] d[i]=d[k]$ for $0 \leq$ $k \leq m-1$ and 0 otherwise is a time-limited sequence with the prescribed lowpass DFT coefficients.

In the next section, we develop a method for constructing signals with prescribed properties in both $\mathcal{W}$ and $\mathcal{S}$. Using this method, we can generalize our construction here to produce a signal with prescribed lowpass coefficients and prescribed values on a given time interval.

\section{Constructing Signals With Prescribed Properties IN $\mathcal{S}$ AND $\mathcal{W}$}

Suppose now we wish to construct $f$ with prescribed properties in two disjoint spaces $\mathcal{W}$ and $\mathcal{S}$, so that $\left\langle s_{i}, f\right\rangle=c[i]$ and $\left\langle w_{i}, f\right\rangle=d[i]$, where the vectors $s_{i}$ and $w_{i}$ form Riesz bases for $\mathcal{S}$ and $\mathcal{W}$, respectively.

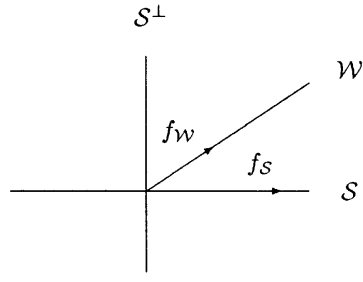

(a) (b)

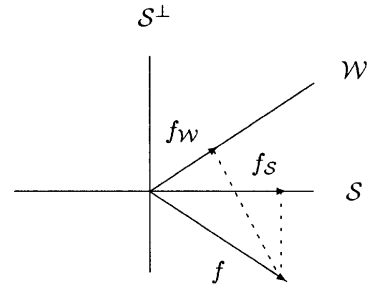

Fig. 2. Illustration of the construction of $f$ with given orthogonal projections $f_{\mathcal{S}}$ onto $\mathcal{S}$ and $f_{\mathcal{W}}$ onto $\mathcal{W}$ with $\mathcal{W} \cap \mathcal{S}=\{0\}$. (a) Desired orthogonal projections onto $\mathcal{S}$ and $\mathcal{W}$. (b) Unique signal $f \in \mathcal{U}=\mathcal{W} \oplus \mathcal{S}$ with the given projections.

To construct $f$, we can define the combined basis $\left\{t_{i}\right\}$ for $\mathcal{W} \oplus \mathcal{S}$, consisting of the vectors $\left\{s_{i}\right\}$ and $\left\{w_{i}\right\}$. Then, with $T$ denoting the set transformation corresponding to the vectors $t_{i}$

$$
f=T\left(T^{*} T\right)^{-1} a
$$

where $a$ is the concatenation of $c$ and $d$.

In what follows, we use the results and insight developed in the previous sections to derive an alternative construction, that although is mathematically equivalent to (3), provides further insight into the problem. The advantage of this approach is that in many cases $f$ can be constructed simply by inspection, without having to formally employ (3), as we demonstrate in the context of a concrete example in Section V.

In analogy to the previous problem, constructing $f$ with $\left\langle s_{i}, f\right\rangle=c[i]$ and $\left\langle w_{i}, f\right\rangle=d[i]$ is equivalent to constructing $f$ to have a prescribed orthogonal projection $f_{\mathcal{S}}$ onto $\mathcal{S}$ and a prescribed orthogonal projection $f_{\mathcal{W}}$ onto $\mathcal{W}$. Fig. 2(a) depicts the orthogonal projections of an unknown signal $f$ onto $\mathcal{S}$ and $\mathcal{W}$. The problem then is to construct $f$ with these projections. With $\mathcal{U}=\mathcal{W} \oplus \mathcal{S}$, it is obvious that $f$ can be arbitrary on $\mathcal{U}^{\perp}$. However, there is a unique $f \in \mathcal{U}$ compatible with the given projections; this vector is illustrated in Fig. 2(b). Fig. 2 illustrates that with $\mathcal{W} \cap \mathcal{S}=\{0\}$, we can always construct $f$ with the desired properties. Furthermore, $P_{\mathcal{U}} f$ is unique.

To explicitly construct the unique $f \in \mathcal{U}$ satisfying the required constraints, we write $f$ as $f=s+v$ where $s \in \mathcal{S}$ and $v \in \widetilde{\mathcal{S}}=\mathcal{S}^{\perp} \cap \mathcal{U}$. Then, since $\left\langle s_{i}, f\right\rangle=\left\langle s_{i}, s\right\rangle$, constructing $f$ such that $\left\langle s_{i}, f\right\rangle=c[i]$ is equivalent to constructing $s \in \mathcal{S}$ such that $\left\langle s_{i}, s\right\rangle=c[i]$. From Theorem $1, s=S\left(S^{*} S\right)^{-1} c$, so that our problem reduces to finding $v \in \widetilde{\mathcal{S}}$ such that $\left\langle w_{i}, v\right\rangle=$ $d[i]-\left\langle w_{i}, s\right\rangle \triangleq d^{\prime}[i]$, which is equivalent to the problem of Section II. Since the orthogonal complement $\widetilde{\mathcal{S}}^{\perp}$ of $\widetilde{\mathcal{S}}$ in $\mathcal{U}$ is equal to $\mathcal{S}, \widetilde{\mathcal{S}}^{\perp} \cap \mathcal{W}=\{0\}$, and we can apply (2) to obtain $v=$ $V\left(W^{*} V\right)^{-1} d^{\prime}=V\left(W^{*} V\right)^{-1}\left(d-W^{*} s\right)$, where $V$ corresponds to a basis for $\widetilde{\mathcal{S}}$. Finally, the unique $f \in \mathcal{U}$ satisfying the desired constraints is

$$
f=S\left(S^{*} S\right)^{-1} c+V\left(W^{*} V\right)^{-1}\left(d-W^{*} S\left(S^{*} S\right)^{-1} c\right) .
$$

Note that there are many alternative methods of constructing $f$. For example, instead of utilizing the decomposition $f=s+v$ we can decompose $f$ as $f=x+v$ where $v \in \widetilde{\mathcal{S}}$ and $x$ is in a subspace $\mathcal{X}$ such that $\mathcal{X} \oplus \widetilde{\mathcal{S}}=\mathcal{U}$. We then construct $f$ by first finding the unique vector $x \in \mathcal{X}$ such that $\left\langle s_{i}, x\right\rangle=c[i]$, and then finding the unique $v \in \widetilde{\mathcal{S}}$ such that $\left\langle w_{i}, v\right\rangle=d[i]-$ $\left\langle w_{i}, x\right\rangle$. We may also exchange the roles of $\mathcal{S}$ and $\mathcal{W}$ and utilize 

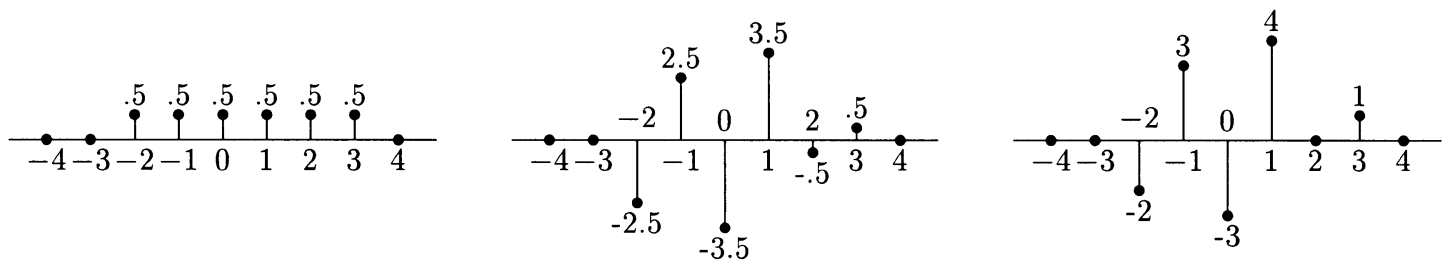

Fig. 3. Constructing a sequence $f$ with prescribed local averages and prescribed odd part. (a) Unique $f_{1} \in \mathcal{S}$ with required local averages. (b) Unique $f_{2} \in \mathcal{S}^{\perp}$ with odd part equal to the difference between the required odd part and the odd part of $f_{1}$. (c) Unique $f=f_{1}+f_{2}$ with both the required local averages and the required odd part.

a decomposition of the form $f=w+y$ where now $w \in \mathcal{W}$ and $y \in \mathcal{W}^{\perp} \cap \mathcal{U}$.

\section{Constructing a Signal With Prescribed Local AVERAGES AND PRESCRIBED OdD PART}

As an illustration of our approach, suppose we want to construct $f$ with local averages $f[2 k]+f[2 k+1]=c[k]$, where $c[-1]=c[0]=c[1]=1$ and $c[k]=0$ otherwise, and with odd part $f[k]-f[-k]=d[k]$, where $d[1]=1, d[2]=2, d[3]=1$, and $d[k]=0, k \geq 4$.

In this example, $\mathcal{S}$ is the subspace of signals $x$ that satisfy $x[2 k]=x[2 k+1]$ for all $k$, and $\mathcal{W}$ is the subspace of odd signals. Using our general method described in the previous section, we can construct the signal $f$ by inspection, as illustrated in Fig. 3. Fig. 3(a) depicts the unique $f_{1} \in \mathcal{S}$ with the desired local averages, so that $f_{1}[2 k]+f_{1}[2 k+1]=c[k]$. Fig. 3(b) depicts the unique $f_{2} \in \mathcal{S}^{\perp}$ with odd part satisfying $f_{2}[k]-f_{2}[-k]=d[k]-x[k]$, where $x$ is the odd part of $f_{1}$. Note that, as we expect, the local averages of $f_{2}$ are zero. Fig. 3(c) depicts $f=f_{1}+f_{2}$, which is the unique sequence with the desired local averages and the desired odd part.

\section{CONCLUSION}

In this letter, we derived general methods for constructing signals with prescribed properties and illustrated the methods in the context of concrete examples. The methods we developed allow for almost arbitrary constraint and construction spaces, and are based on a geometric interpretation. We considered both the problem of constructing a signal in a subspace $\mathcal{W}$ with prescribed properties associated with a subspace $\mathcal{S}$, as well as the problem of constructing a signal with prescribed properties in both $\mathcal{W}$ and $\mathcal{S}$. The latter construction can be extended in a straightforward way to the case in which properties in more than two subspaces are considered.

\section{ACKNOWLEDGMENT}

The author wishes to thank the anonymous reviewers for their constructive comments.

\section{REFERENCES}

[1] R. M. Young, An Introduction to Nonharmonic Fourier Series. New York: Academic, 1980.

[2] Y. C. Eldar, "Quantum signal processing," Ph.D. thesis, Massachusetts Inst. Technol., Cambridge, MA, Dec., 2001. Available [Online]: http://allegro.mit.edu/dspg/publications/Tech Rep/index.html.

[3] M. Unser and A. Aldroubi, "A general sampling theory for nonideal acquisition devices," IEEE Trans. Signal Processing, vol. 42, pp. 2915-2925, Nov. 1994.

[4] Y. C. Eldar, "Sampling without input constraints: Consistent reconstruction in arbitrary spaces," in Sampling, Wavelets and Tomography, A. Zayed and J. J. Benedetto, Eds., to be published.

[5] - "Sampling and reconstruction in arbitrary spaces and oblique dual frame vectors," J. Fourier Analys. Appl., vol. 1, no. 9, pp. 77-96, Jan. 2003. 